\title{
Synthesis of deuterium-labelled amlexanox and its metabolic stability against mouse, rat and human microsomes
}

Xinmin Gan ${ }^{\mathrm{a}, \mathrm{b}}$, Michael W. Wilson ${ }^{\mathrm{a}, \mathrm{b}}$, Tyler S. Beyett ${ }^{\mathrm{c}, \mathrm{d}}$, Bo Wen ${ }^{\mathrm{e}}$, Duxin Sun ${ }^{\mathrm{e}}$, Scott D. Larsen $^{\mathrm{a}, \mathrm{b}}$, John J. G. Tesmer ${ }^{\mathrm{f}}$, Alan R. Saltiel ${ }^{\mathrm{g}}$ and Hollis D. Showalter ${ }^{\mathrm{a}, \mathrm{b}^{*}}$

${ }^{\mathrm{a}}$ Department of Medicinal Chemistry, ${ }^{\mathrm{b}}$ Vahlteich Medicinal Chemistry Core, ${ }^{\mathrm{c}}$ Program in Chemical Biology, ${ }^{\mathrm{d}}$ Life Sciences Institute, Departments of Pharmacology and Biological Chemistry, ${ }^{e}$ Department of Pharmaceutical Sciences, University of Michigan, Ann Arbor, MI 48109, United States

${ }^{\mathrm{f}}$ Departments of Biological Sciences and Medicinal Chemistry and Molecular Pharmacology, Purdue University, West Lafayette, IN 47907-2054

${ }^{\mathrm{g}}$ Departments of Medicine and Pharmacology, Institute for Diabetes and Metabolic Health, University of California, San Diego, La Jolla, CA 92093-0912, United States

*Corresponding author

\begin{abstract}
As part of a program toward making analogues of amlexanox (1), currently under clinical investigation for the treatment of type 2 diabetes and obesity, we have synthesized derivative $\mathbf{5}$ in which deuterium has been introduced into two sites of metabolism on the C-7 isopropyl function of amlexanox. The synthesis of $\mathbf{5}$ was completed in an efficient three-step process utilizing reduction of key olefin $\mathbf{7 b}$ to $\mathbf{8}$ by Wilkinson's catalyst to provide specific incorporation of di-deuterium across the double bond. Compound $\mathbf{5}$ displayed nearly equivalent potency to amlexanox ( $\mathrm{IC}_{50} 1.1 \mu \mathrm{M}$ vs $0.6 \mu \mathrm{M}$, respectively) against recombinant human $\mathrm{TBK} 1$. When incubated with human, rat, and mouse liver microsomes, amlexanox (1) and $d_{2}$-amlexanox

This is the author manuscript accepted for publication and has undergone full peer review but has not been through the copyediting, typesetting, pagination and proofreading process, which may lead to differences between this version and the Version of Record. Please cite this article as doi: $10.1002 /$ jlcr.3716
\end{abstract}

This article is protected by copyright. All rights reserved. 
(5) were stable $\left(\mathrm{t}_{1 / 2}>60 \mathrm{~min}\right)$ with $\mathbf{1}$ showing marginally greater stability relative to 5 except for rat liver microsomes. These data show that incorporating deuterium into two sites of metabolism does not majorly suppress Cyp-mediated metabolism relative to amlexanox.

Keywords: obesity, amlexanox, $d_{2}$-amlexanox, metabolites, microsomal stability, TBK1 kinase, Wilkinson's catalyst

\section{INTRODUCTION}

This article is protected by copyright. All rights reserved. 
There is currently a worldwide epidemic of obesity, which is manifesting itself as a leading risk factor in industrialized countries for the development of type 2 diabetes, dyslipidaemia, nonalcoholic fatty liver disease, cardiovascular disease and some cancers. ${ }^{1}$ Obesity results from a sustained imbalance between calorie intake and energy expenditure and is characterized by chronic, low-grade inflammation in liver and adipose tissues, which in turn attenuates the actions of multiple hormones to produce a dysregulated metabolic state. ${ }^{2,3,4}$ Prior studies on the role of inflammation in the generation of insulin resistance and type 2 diabetes during obesity implicate an important role for the nuclear factor kappa-light-chain-enhancer of activated B cells (NF- $\kappa B$ )

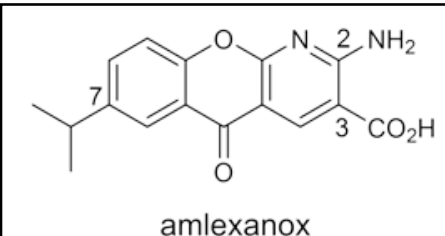

(1)

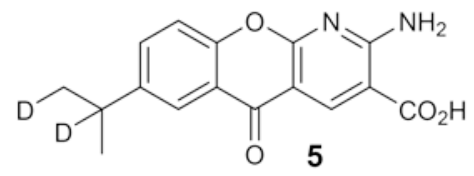

Figure 1. Structures of amlexanox (1), metabolites (2 - 4) and

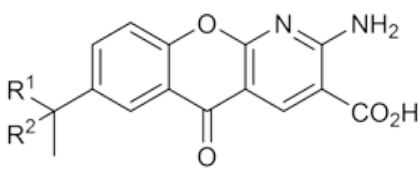

2: $\mathrm{R}^{1}=\mathrm{CH}_{3} ; \mathrm{R}^{2}=\mathrm{OH}$

3: $\mathrm{R}^{1}=\mathrm{CH}_{2} \mathrm{OH} ; \mathrm{R}^{2}=\mathrm{H}$

4: $\mathrm{R}^{1}=\mathrm{CO}_{2} \mathrm{H} ; \mathrm{R}^{2}=\mathrm{H}$

pathway, revealing the role of two noncanonical inhibitors of kappa B (IкB) kinases, TANK-binding kinase 1 (TBK1) and inhibitor of nuclear factor kappa-B kinase subunit $\varepsilon(\mathrm{IKK} \varepsilon)$, in insulin-independent pathways that promote energy storage and block adaptive energy expenditure during obesity. ${ }^{5,6}$ Since none of the currently available and widely used treatments for obesity addresses the underlying energy imbalance associated with inflammation pathways, ${ }^{7}$ we initiated a high throughput screen for small molecule inhibitors of the TBK1 and IKKe kinases. One hit from our screen was amlexanox (1; Figure 1), an approved drug developed by Takeda that is used for the treatment of canker sores, asthma, and allergic rhinitis. ${ }^{8,9}$ The compound is a modest inhibitor of TBK1 and IKK $\varepsilon$ and reproduced the effects of the IKK $\varepsilon$ knockout phenotype, which include weight loss in obese mice, improved insulin sensitivity, and reduced hepatic steatosis and inflammation. Amlexanox also increased the level of the second messenger molecule cAMP and triggered the release of the hormone interleukin-6 (IL-6) from fat cells thereby reducing the production of glucose. Two recent clinical trials indicated that amlexanox treatment effectively lowered glycated hemoglobin 
(HbA1c) in a subset of patients with obesity and type 2 diabetes. ${ }^{10}$ Despite these promising results, the future utility of amlexanox in the clinic for obesity and type 2 diabetes intervention may be limited by its solubility, modest potency, and/or metabolic profile.

Previous studies by Takeda investigators showed that the oral administration of amlexanox (1) in mice, rats, guinea pigs, and dogs generated in plasma a major mono-hydroxyl metabolite (2) from oxidation of the C-7 isopropyl substituent, and two minor metabolites 3 and $\mathbf{4}^{11,12}$ (Figure 1). The glucuronide of $\mathbf{1}$ was also identified in rats. In humans, the main metabolite was $\mathbf{2}$. The structures of metabolites $\mathbf{2}$ and $\mathbf{3}$ have been confirmed by synthesis. ${ }^{13}$

As part of a program toward making analogues of amlexanox, ${ }^{14,15}$ we decided to synthesize a derivative 5 (Figure 1) in which deuterium is introduced into two sites of metabolism on the C-7 isopropyl function. The deuterium kinetic isotope effect associated with placing deuterium at the site of metabolic derivatization slows metabolic activation and thus is expected to increase the lifetime of the active drug in vivo. ${ }^{16}$ Herein we provide data of the activity of $\mathbf{5}$ relative to amlexanox (1) in inhibiting TBK1 and its metabolic stability against mouse, rat and human microsomes.

\section{RESULTS AND DISCUSSION}

Synthetic chemistry. Initial approaches toward generating $d_{1}$-amlexanox, in which the benzylic C-7 methine was exchanged for deuterium, centered on

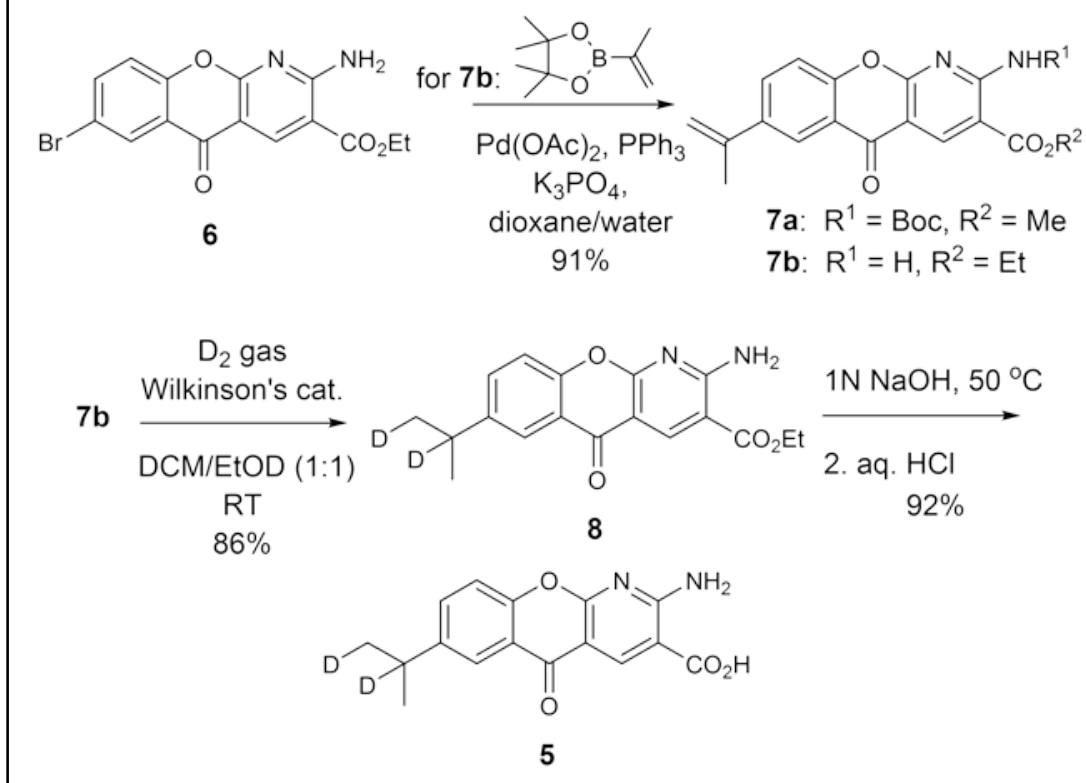

Scheme 1. Synthesis of $d_{2}$-amlexanox (5) 
catalytic exchange chemistry. Thus, reaction of amlexanox (1) under a variety of conditions (hydrogen gas, $10-30 \%$ palladium on activated carbon, methanol- $d_{4}, 25-50{ }^{\circ} \mathrm{C}$ ) left starting material only. We then focused on incorporation of deuterium through room temperature hydrogenation of the olefin in compound 7a, made by known procedures, ${ }^{13,17}$ with deuterium gas in the presence of $10 \%$ palladium on activated carbon in ethyl acetate. Thus, a control reduction of 7a with hydrogen gave cleanly the non-deuterated C-7 isopropyl product. Substitution of deuterium for hydrogen gas yielded a new set of products with mass spectrum peaks associated with incorporation of more than two atoms of deuterium. The ${ }^{1} \mathrm{H}$ NMR spectrum suggested that the predominant $N$-Boc carboxylic methyl ester product had incorporated four atoms of deuterium and ${ }^{13} \mathrm{C}$ NMR experiments on the next step hydrolysis product (analogous to 5) indicated the presence of seven uniquely substituted C-7 methine carbons. This showed that deuterium had been incorporated non-specifically into both C-7 methyl groups as well as the methine carbon. There is literature precedence for such scrambling of isotopic incorporation into benzylic and allylic sites. ${ }^{18}$

After unsuccessfully surveying other heterogenous catalytic conditions, we then resorted to olefin reduction with a homogeneous catalyst (Scheme 1). Employing our recently published methodology of introducing C-7 substituents via late-stage coupling chemistry to make amlexanox analogues ${ }^{15}$, we reacted tricyclic bromide $6^{19}$ with 2-propenyl pinacol boronic ester under standard Suzuki coupling conditions to give $\mathbf{7 b}$ in $91 \%$ yield. Reaction with tris(triphenylphosphine)rhodium(I) chloride (Wilkinson's catalyst) and deuterium gas, as previously described for similar reductions, ${ }^{20,21}$ proceeded in high yield to give key intermediate $\mathbf{8}$ with specific incorporation of di-deuterium across the double bond. Standard hydrolysis conditions as described before ${ }^{15}$ then provided $d_{2}$-amlexanox (5) in $92 \%$ yield and $72 \%$ overall yield from starting ester $\mathbf{6}$. All compounds were rigorously purified by

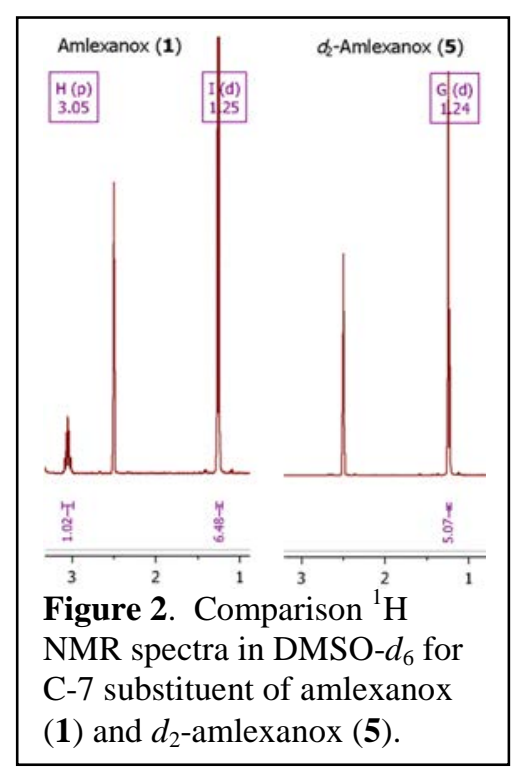


flash chromatography or crystallization, and their structural assignments are supported by diagnostic peaks in the ${ }^{1} \mathrm{H}$ and ${ }^{13} \mathrm{C}$ NMR spectra and by high resolution mass spectrometry. Digital copies of NMR and mass spectra can be found in Supplementary Materials Online.

The ${ }^{1} \mathrm{H}$ NMR spectra of the upfield region ( $\delta 1-3 \mathrm{ppm}$ ) for the C-7 isopropyl substituent (Figure 2) clearly show clean, specific incorporation of di-deuterium into 5. Complete collapse of the symmetrical pentet at $\delta 3.05$ ppm occurs in 1 and the symmetrical doublet at $\delta 1.25 \mathrm{ppm}$, integrating for six protons, changes to an unsymmetrical doublet in $\mathbf{5}$ integrating for five protons. High resolution mass spectra for $\mathbf{5}$ and $\mathbf{8}$ (Supplementary Materials Online) also support specific

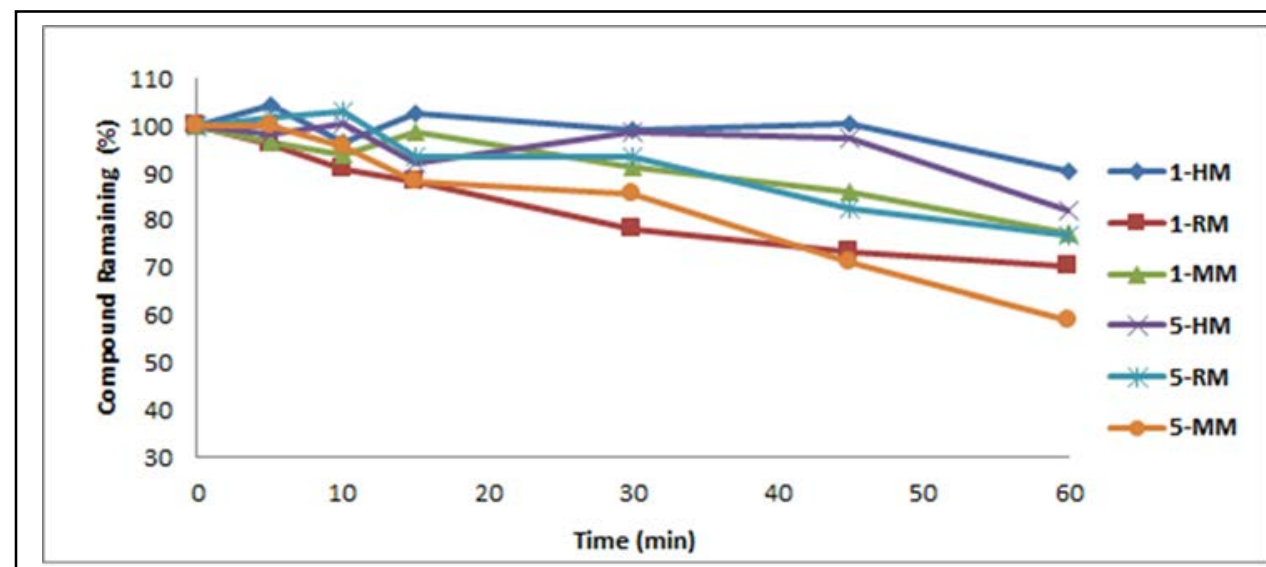

Figure 3. Human $(\mathrm{H})$, rat $(\mathrm{R})$ and mouse $(\mathrm{M})$ microsomal stability for amlexanox (1) and $d_{2}$-amlexanox (5) incorporation of di-deuterium.

$\underline{\text { Metabolic }}$ stability studies. Amlexanox (1) and $d_{2}$-amlexanox (5) are moderately metabolized by

human, rat and mice liver microsomes. The in vitro disappearance half-life $\left(\mathrm{t}_{1 / 2}\right)$ of both compounds in all three microsomes is over $60 \mathrm{~min}$ (Figure 3). During the incubation in human and mice liver microsomes, about $8 \%$ more unmetabolized amlexanox remains after $60 \mathrm{~min}$ compared to $d_{2}$-amlexanox. However, $d_{2}$-amlexanox (5) is more stable than amlexanox (1) in rat liver microsomes. Both compounds show greater stability in human liver microsomes relative to the other microsomes. Verapamil was employed as positive control compound in order to ensure that the microsomes and NADPH used in this study were active and that incubation conditions 
were appropriate to perform the studies.

Based on the observed degradation

patterns, amlexanox (1) and $d_{2^{-}}$

amlexanox (5) are considered to be

stable against the three liver

microsomes.

Activity against TBK1 kinase. $d_{2^{-}}$

Amlexanox (5) was investigated for

inhibition of recombinant human TBK1

as described previously. ${ }^{15}$ The $\mathrm{IC}_{50}$

values shown were determined by dose-

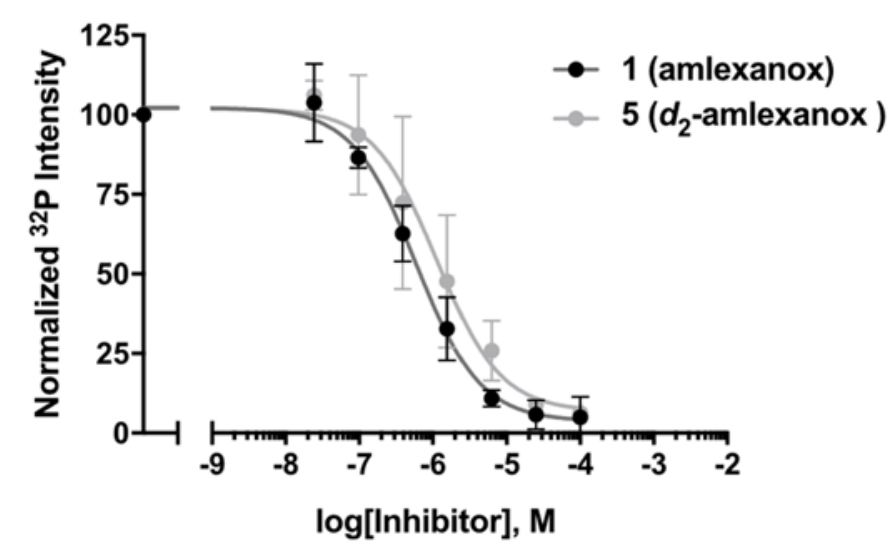

Figure 4. In vitro potency of amlexanox (1) and $d_{2^{-}}$ amlexanox (5) against purified TBK1 as determine via radiometric kinase assay measuring phosphorylation of the substrate myelin basic protein. The goodness of fit $\left(\mathrm{r}^{2}\right)$ for $\mathbf{1}$ and $\mathbf{5}$ is 0.97 and 0.91 , respectively, with no statistically significant difference via Student's two-tailed T-test $(n=4$, reported as mean $\pm \mathrm{SD}$ ).

response radiometric kinase assays with

myelin basic protein as substrate, and are technically apparent $\mathrm{IC}_{50}$ values. Each is an average of three separate determinations. Since the isopropyl

group on amlexanox is solvent exposed, ${ }^{14}$ incorporation of deuterium is not expected to affect inhibitor potency. Indeed, the in vitro potencies of amlexanox (1) and $d_{2}$-amlexanox (5) against TBK1 are $0.6 \pm 0.1$ and $1.1 \pm 0.3 \mu \mathrm{M}$, respectively (Figure 4 and Supplementary Materials Online). The difference is not statistically significant demonstrating that amlexanox can be deuterated without affecting inhibitor potency.

\section{CONCLUSIONS}

As part of a program toward making analogues of amlexanox (1), which is being evaluated clinically for the treatment of type 2 diabetes and obesity, we decided to synthesize a derivative 5 to determine if placing deuterium at two sites of known metabolic activation of amlexanox might increase its half-life against three species of liver microsomes. Microsomes are rich in cytochrome (Cyp) P-450 enzymes, which are the major enzymes involved in drug metabolism 
and account for about $75 \%$ of total metabolism. ${ }^{22}$ The most common pathway of Cyp-mediated metabolism is hydroxylation of aliphatic carbons.

The data in Figure 3 show that amlexanox (1) and $d_{2}$-amlexanox (5) are relatively stable against all three liver microsomes $\left(\mathrm{t}_{1 / 2}>60 \mathrm{~min}\right)$ with amlexanox showing marginally greater stability relative to $d_{2}$-amlexanox except for rat liver microsomes. Hence, blocking two sites of metabolism with incorporation of a single deuterium on the C-7 methine carbon and another on a flanking methyl group of $\mathbf{5}$ does not majorly suppress Cyp-mediated metabolism relative to amlexanox. Metabolite identification experiments will be required to expand upon these findings. Additionally, synthetic chemistry needs to be developed that can specifically incorporate C-7 deuterium patterns (e.g., - $\mathrm{CH}\left(\mathrm{CH}_{3}\right)\left(\mathrm{CH}_{2} \mathrm{D}\right)$ and $\left.-\mathrm{CD}\left(\mathrm{CH}_{3}\right)_{2}\right)$ aligned with known metabolites 2-4, and additional patterns (e.g., - $\left.\mathrm{CH}\left(\mathrm{CD}_{3}\right)_{2},-\mathrm{CD}\left(\mathrm{CD}_{3}\right)_{2}\right)$ to more fully explore the deuterium isotope effect.

\section{EXPERIMENTAL}

4.1 Materials and instrumentation. All starting monomers were obtained from commercial suppliers and were used without further purification. Routine ${ }^{1} \mathrm{H}$ NMR spectra were recorded at 400 or $500 \mathrm{MHz}$ on a Varian 400 or 500 instrument, respectively, with $\mathrm{CDCl}_{3}$ or DMSO- $d_{6}$ as solvent. Chemical shift values are recorded in $\delta$ units (ppm). High resolution mass spectrometry (HRMS) analysis was performed on an Agilent Q-TOF system. Analytical HPLC was performed on an Agilent 1100 series instrument with an Agilent Zorbax Eclipse Plus C18 (4.6 mm $\times 75$ $\mathrm{mm}, 3.5 \mu \mathrm{m}$ particle size) column with the gradient $10 \%$ acetonitrile/water $(1 \mathrm{~min}), 10-90 \%$ acetonitrile/water $(6 \mathrm{~min})$, and $90 \%$ acetonitrile/water $(2 \mathrm{~min})$ flow $=1 \mathrm{~mL} / \mathrm{min}$. Thin-layer chromatography (TLC) was performed on silica gel GHLF plates $(250 \mu \mathrm{m})$ purchased from Analtech. Column chromatography was carried out in the flash mode utilizing silica gel 
(220-240 mesh) purchased from Silicycle. Extraction solutions were dried over anhydrous sodium sulfate prior to concentration.

\subsection{Ethyl 2-amino-5-oxo-7-(prop-1-en-2-yl)-5H-chromeno[2,3-b]pyridine-3-carboxylate}

(7b). A solution of tripotassium phosphate $(2.63 \mathrm{~g}, 12.4 \mathrm{mmol})$ in water $(6 \mathrm{~mL})$ was added to a nitrogen-degassed suspension of ethyl 2-amino-7-bromo-5-oxo-5H-chromeno[2,3b]pyridine-3-carboxylate ${ }^{19}$ (6; $750 \mathrm{mg}, 2.07 \mathrm{mmol}$ ), 4,4,5,5-tetramethyl-2-(prop-1-en-2-yl)1,3,2-dioxaborolane ( $833 \mathrm{mg}, 4.96 \mathrm{mmol}$ ), triphenylphosphine (271 mg, $1.03 \mathrm{mmol})$, $\mathrm{Pd}(\mathrm{OAc})_{2}(60.3 \mathrm{mg}, 0.27 \mathrm{mmol})$ in $p$-dioxane $(60 \mathrm{~mL})$ in a $500 \mathrm{~mL} \mathrm{RB}$ flask. The mixture was heated at $100-105^{\circ} \mathrm{C}$ under nitrogen for $2 \mathrm{~h}$. After cooling, the mixture was diluted with water $(50 \mathrm{~mL})$ and extracted with dichloromethane $(2 \times 120 \mathrm{~mL})$. The combined organic phases were dried $\left(\mathrm{Na}_{2} \mathrm{SO}_{4}\right)$ and concentrated to leave an orange solid that was triturated sequentially with small volumes of ethanol and ethyl ether, collected, and dried to leave $7 \mathbf{b}$ (610 mg, 91\%) as a white solid: mp $245-247{ }^{\circ} \mathrm{C} .{ }^{1} \mathrm{H}$ NMR (400 MHz, chloroformd): $\delta 9.16(\mathrm{~s}, 1 \mathrm{H}), 8.38(\mathrm{~s}, 1 \mathrm{H}), 8.31(\mathrm{~d}, J=2.3 \mathrm{~Hz}, 1 \mathrm{H}), 7.86(\mathrm{dd}, J=8.8,2.4 \mathrm{~Hz}, 1 \mathrm{H}), 7.47$ (d, $J=8.7 \mathrm{~Hz}, 1 \mathrm{H}), 5.86(\mathrm{~s}, 1 \mathrm{H}), 5.49(\mathrm{~d}, J=1.3 \mathrm{~Hz}, 1 \mathrm{H}), 5.23-5.18(\mathrm{~m}, 1 \mathrm{H}), 4.41(\mathrm{q}, J=$ $7.1 \mathrm{~Hz}, 2 \mathrm{H}), 2.24(\mathrm{dd}, J=1.5,0.8 \mathrm{~Hz}, 3 \mathrm{H}), 1.45(\mathrm{t}, J=7.1 \mathrm{~Hz}, 3 \mathrm{H}) . \mathrm{HRMS}: m / z(\mathrm{M}+\mathrm{H})^{+}$: calcd. for $\mathrm{C}_{18} \mathrm{H}_{16} \mathrm{~N}_{2} \mathrm{O}_{4}$ : 325.1183; Found, 325.1188.

\subsection{Ethyl 2-amino-5-oxo-7-(propan-2-yl-1,2- $\left.d_{2}\right)$-5H-chromeno[2,3-b]pyridine-3-carboxylate} (8). Tris(triphenylphosphine)rhodium(I) chloride (Wilkinson's catalyst; $339 \mathrm{mg}, 0.366$ mmol) was completely dissolved in anhydrous dichloromethane $(10 \mathrm{~mL})$ and ethanol- $d_{1}(10$ $\mathrm{mL}$ ) in a $250 \mathrm{~mL}$ 3-neck RB flask under nitrogen. The flask with the resulting clear red brown solution was evacuated by a water pump and then flushed with deuterium gas in a balloon. This flushing/evacuation procedure was repeated three more times, and then the mixture was stirred under deuterium gas (balloon pressure) for $20 \mathrm{~min}$ at room temperature. Then compound 7 (360 mg, $1.11 \mathrm{mmol}$ ) dissolved in a mixture of warm dichloromethane 
(75 mL) and ethanol- $d_{1}(75 \mathrm{~mL})$ was added by syringe. The resulting solution was stirred at room temperature for $4.5 \mathrm{~h}$ and then concentrated to a solid that was triturated in a small volume of benzene. The collected solids were further purified by flash silica gel column chromatography (elution with $0.5-1.0 \%$ methanol in dichloromethane). Product fractions were combined and concentrated to leave 8 (315 $\mathrm{mg}, 86 \%)$ as a while solid: $\mathrm{mp} 239-241{ }^{\circ} \mathrm{C}$. ${ }^{1} \mathrm{H}$ NMR (400 MHz, chloroform- $d$ ): $\delta 9.16(\mathrm{~s}, 1 \mathrm{H}), 8.34(\mathrm{~s}, 1 \mathrm{H}), 8.11(\mathrm{~d}, J=2.3 \mathrm{~Hz}, 1 \mathrm{H})$, $7.59(\mathrm{dd}, J=8.6,2.3 \mathrm{~Hz}, 1 \mathrm{H}), 7.44$ (d, $J=8.6 \mathrm{~Hz}, 1 \mathrm{H}), 5.84$ (s, 1H), 4.41 (q, $J=7.1 \mathrm{~Hz}$, 2H), $1.44(\mathrm{t}, J=7.1 \mathrm{~Hz}, 3 \mathrm{H}), 1.30(\mathrm{~d}, J=6.5 \mathrm{~Hz}, 5 \mathrm{H})$. HRMS: $m / z(\mathrm{M}+\mathrm{H})^{+}$Calcd. for $\mathrm{C}_{18} \mathrm{H}_{16} \mathrm{D}_{2} \mathrm{~N}_{2} \mathrm{O}_{4}$ : 329.1465; Found 329.1480.

\subsection{2-Amino-5-oxo-7-(propan-2-yl-1,2- $\left.d_{2}\right)-5 H$-chromeno[2,3-b]pyridine-3-carboxylic acid} $\left(\boldsymbol{d}_{\mathbf{2}}\right.$-amlexanox; 5). To a suspension of $8(460 \mathrm{mg}, 1.4 \mathrm{mmol})$ in ethanol $(80 \mathrm{~mL})$ and water $(10 \mathrm{~mL})$ was added $1 \mathrm{~N}$ aq. $\mathrm{NaOH}(10 \mathrm{~mL})$ and the mixture was stirred at $50{ }^{\circ} \mathrm{C}$ for $90 \mathrm{~min}$. TLC (5\% methanol in dichloromethane) showed consumption of starting material. The mixture was filtered and the filtrate concentrated to a wet solid that dissolved in water (50 $\mathrm{mL}$ ). The solution was acidified with $1 \mathrm{~N}$ aq. $\mathrm{HCl}$ to $\mathrm{pH} \sim 3$ and the precipitated solids were stirred at room temperature for $20 \mathrm{~h}$. After storage at room temperature for another $24 \mathrm{~h}$, the solids were collected, washed with water, and dried under vacuum at $60{ }^{\circ} \mathrm{C}$ for $4 \mathrm{~h}$ to leave 5 (385 mg, 92\%) as an off-white solid: $\mathrm{mp}>300{ }^{\circ} \mathrm{C}$ (dec.). ${ }^{1} \mathrm{H}$ NMR (500 MHz, DMSO- $\left.d_{6}\right): \delta$ 13.45 (s, 1H), 8.80 (s, 1H), 8.25 (b, 2H), 7.92 (d, $J=2.3 \mathrm{~Hz}, 1 \mathrm{H}), 7.73$ (dd, $J=8.6,2.4 \mathrm{~Hz}$, $1 \mathrm{H}), 7.53(\mathrm{~d}, J=8.6 \mathrm{~Hz}, 1 \mathrm{H}), 1.24(\mathrm{~d}, \mathrm{~J}=8.6 \mathrm{~Hz}, 5 \mathrm{H}) .{ }^{13} \mathrm{C}$ NMR $\left(101 \mathrm{MHz}\right.$, DMSO- $\left.d_{6}\right): \delta$ $174.84,167.35,162.25,161.46,153.02,144.93,141.35,133.71,122.44,120.91,118.04$, 106.23, 105.94, 33.70 - $31.07(\mathrm{~m}), 23.64,23.58$ - 23.11 (m). HRMS: $m / z(\mathrm{M}+\mathrm{H})^{+}$Calcd. for $\mathrm{C}_{16} \mathrm{H}_{12} \mathrm{D}_{2} \mathrm{~N}_{2} \mathrm{O}_{4}$ : 301.1152; Found 301.1157. HPLC: $\mathrm{t}_{\mathrm{R}}, 6.42$, purity $98.3 \%$

4.5 Metabolic stability studies. The in vitro metabolism study of amlexanox and $d_{2^{-}}$ amlexanox were performed in human, rat and mice liver microsomes to evaluate the potential of 
each compound for cytochrome P450-mediated metabolism. The metabolic stability was assessed as described using pooled CD-1 mouse liver microsomes, pooled SD rat liver microsmes and pooled human liver microsomes (purchased from Xeno Tech). $1 \mu \mathrm{M}$ of each compound was incubated with $0.5 \mathrm{mg} / \mathrm{mL}$ liver microsomes and $1.7 \mathrm{mM}$ cofactor $\beta$-NADPH in $0.1 \mathrm{M}$ phosphate buffer $(\mathrm{pH}=7.4)$ containing $3.3 \mathrm{mM} \mathrm{MgCl}_{2}$ at $37{ }^{\circ} \mathrm{C}$. The DMSO concentration was less than $0.1 \%$ in the final incubation mixture. At $0,5,10,15,30,45$, and 60 min of incubation, $40 \mu \mathrm{L}$ of reaction mixture were removed and the reaction stopped immediately by adding 3-fold excess of cold acetonitrile containing $100 \mathrm{ng} / \mathrm{mL}$ of internal standard for quantification. The incubation without the addition of NADPH was used as a negative control. Verapamil were incubated similarly as a positive control substrate. The collected fractions were centrifuged at $15000 \mathrm{rpm}$ for $10 \mathrm{~min}$ to collect the supernatant for LC-MS/MS analysis, from which the amount of remaining compound was determined. The natural log of the amount of compound remaining was plotted against time to determine the disappearance rate and the half-life of tested compounds. The LC-MS/MS method consisted of a Shimadzu HPLC system and chromatographic separation of tested compound was achieved using a Waters XBridge-C18 column $(5 \mathrm{~cm} \times 4.6 \mathrm{~mm}, 3.5 \mu \mathrm{m})$. An AB Sciex QTrap 5500 mass spectrometer equipped with an electrospray ionization source (Applied Biosystems, Toronto, Canada) in the negative-ion multiple reaction monitoring (MRM) mode for detection. The mobile phases were $0.1 \%$ formic acid in purified water (A) and $0.1 \%$ formic acid in acetonitrile (B). The gradient (B) was held at 10\% (0-0.5 min), increased to $95 \%$ at $2 \mathrm{~min}$, kept at isocratic 95\% B for $1.0 \mathrm{~min}$, and then immediately stepped back down to $10 \%$ for a 2 min reequilibration. Flow rate was set at $1.0 \mathrm{~mL} / \mathrm{min}$. Amlexanox, $d_{2}$-amlexanox and internal standard 4-ASA were detected on the mass spectrometer by using multiple reaction monitoring transitions $297.1 \rightarrow 253.2 \mathrm{~m} / \mathrm{z}, 299.2 \rightarrow 255.1 \mathrm{~m} / \mathrm{z}$ and $152.0 \rightarrow 108.0 \mathrm{~m} / \mathrm{z}$, respectively. Mass spectrometry parameter optimization was performed using an automated quantitative method provided by the manufacturer. 
4.6 Kinase assay. The assay was performed as described previously. ${ }^{15}$ Briefly, $50 \mathrm{nM}$ purified TBK1 was incubated with $5 \mu \mathrm{M}$ myelin basic protein and varying concentrations of inhibitor (from $10 \mu \mathrm{M}$ to $0 \mu \mathrm{M}$ ). Reactions were initiated by the addition of $10 \mu \mathrm{M}$ ATP spiked with $\left[\gamma^{-32} \mathrm{P}\right]-\mathrm{ATP}$ and allowed to proceed for $30 \mathrm{~min}$ at room temperature. After quenching the reactions, radioactive phosphorylated substrate was quantified via phosphorimaging plates. Results were analyzed with GraphPad Prism 7 and fit to an inhibition dose-response sigmoidal curve with the Hill slope constrained to -1. Assays were repeated four independent times and the results reported as mean \pm standard deviation. Statistical significance was determined via Student's two-tailed T-test $(\mathrm{p}<0.05)$.

\section{ACKNOWLEDGMENTS}

This study was supported by National Institutes of Health (NIH) Pharmacological Sciences Training Program fellowship [T32-GM007767] and U.S. Department of Education GAANN fellowship [P200A150164] to TSB, and NIH R01 DK100319 to XG, JJGT, ARS, and HDS.

\section{CONFLICT OF INTEREST}

The authors report no conflicts of interest.

\section{ORCID}

Hollis Showalter; http://orcid.org/0000-0003-2863-0170

\section{REFERENCES}

1. Giordano A, Frontini A, Cinti S. Convertible visceral fat as a therapeutic target to curb obesity. Nat Rev Drug Discovery. 2016;15(6):405-424.

2. Saltiel AR. New therapeutic approaches for the treatment of obesity. Science Translational Medicine. 2016;8(323 323rv2):1-11. 
3. Mowers J, Uhm M, Reilly SM, et al. Inflammation produces catecholamine resistance in obesity via activation of PDE3B by the protein kinases IKK $\varepsilon$ and TBK1. eLife. 2013;2:e01119/01111-01118.

4. Reilly SM, Saltiel AR. Adapting to obesity with adipose tissue inflammation. Nat Rev Endocrinol. 2017;13(11):633-643.

5. Chiang $\mathrm{S}-\mathrm{H}$, Bazuine $\mathrm{M}$, Lumeng $\mathrm{CN}$, et al. The protein kinase $\mathrm{IKK} \varepsilon$ regulates energy balance in obese mice. Cell (Cambridge, MA, US). 2009;138(5):961-975.

6. Saltiel AR. Insulin resistance in the defense against obesity. Cell Metab. 2012;15(6):798804.

7. Clapham JC, Arch JRS. Targeting thermogenesis and related pathways in anti-obesity drug discovery. Pharmacol Ther. 2011;131(3):295-308.

8. Makino H, Saijo T, Ashida Y, Kuriki H, Maki Y. Mechanism of action of an antiallergic agent, amlexanox (AA-673), in inhibiting histamine release from mast cells. Acceleration of cAMP generation and inhibition of phosphodiesterase. Int Arch Allergy Appl Immunol. 1987;82(1):66-71.

9. Bell J. Amlexanox for the treatment of recurrent aphthous ulcers. Clin Drug Invest. 2005;25(9):555-566.

10. Oral EA, Reilly SM, Gomez AV, et al. Inhibition of IKK $\varepsilon$ and TBK1 improves glucose control in a subset of patients with type 2 diabetes. Cell Metab. 2017;26(1):157-170.e157.

11. Torii H, Yoshida K, Tsukamoto T, et al. Metabolic fate of amoxanox (AA-673), a new antiallergic agent, in rats, mice, guinea pigs and dogs. Yakuri to Chiryo. 1985;13(9):4933-4954.

12. Yoshida K, Danayama K. Pharmacokinetics and metabolism of amlexanox (AA-673), a new antiallergic agent, after nasal administration to rats. Yakuri to Chiryo. 1987;15(5):1873-1881.

13. Ukawa K, Ishiguro T, Kuriki H, Nohara A. Synthesis of the metabolites and degradation products of 2-amino-7-isopropyl-5-oxo-5H-[1]benzopyrano[2,3-b]pyridine-3-carboxylic acid (amoxanox). Chem Pharm Bull. 1985;33(10):4432-4437.

14. Beyett TS, Gan X, Reilly SM, et al. Carboxylic acid derivatives of amlexanox display enhanced potency toward TBK1 and IKK $\varepsilon$ and reveal mechanisms for selective inhibition. Mol Pharmacol. 2018;94(4):1210-1219.

15. Beyett TS, Gan X, Reilly SM, et al. Design, synthesis, and biological activity of substituted 2-amino-5-oxo-5H-chromeno[2,3- $b$ ]pyridine-3-carboxylic acid derivatives as inhibitors of the inflammatory kinases TBK1 and IKK $\varepsilon$ for the treatment of obesity. Bioorg Med Chem. 2018;26(20):5443-5461.

16. Shao L, Hewitt MC. The kinetic isotope effect in the search for deuterated drugs. Drug News Perspect. 2010;23(6):398-404.

17. Saltiel AR, Showalter HD, Larsen S. Preparation of deuterated amlexanox derivatives for use as TBK1 or IKKe protein kinase inhibitors. US Patent 9,944,652, Apr. 17, 2018. 
18. Nishimura S. Handbook of Heterogeneous Catalytic Hydrogenation for Organic Synthesis. New York: John Wiley \& Sons; 2001. Chapter 3, pp. 68-72.

19. Ghosh C, Sinharoy DK, Mukhopadhyay KK. Heterocyclic systems. Part 6. Reactions of 4-oxo-4H-[1]benzopyran-3-carbonitriles with hydrazine, phenylhydrazine, hydroxylamine, and some reactive methylene compounds. J Chem Soc, Perkin Trans 1. 1979(8):1964-1968.

20. Rakoff H. Preparation of fatty acids and esters containing deuterium. Prog Lipid Res. 1982;21(3):225-254.

21. Lie Ken Jie MSF, Choi YC. Mass spectral studies of deuterium-labeled picolinyl fatty esters in the determination of double-bond positions. J Am Oil Chem Soc. 1992;69(12):1245-1247.

22. Guengerich FP. Cytochrome P450 and chemical toxicology. Chem Res Toxicol. 2008;21(1):70-83.

\section{SUPPORTING INFORMATION}

Additional Supporting Information may be found online in the supporting information tab for this article $\left({ }^{1} \mathrm{H}\right.$ NMR and high resolution mass spectra data for compounds $\mathbf{1}, \mathbf{5}, \mathbf{7 a}$ and $\mathbf{8},{ }^{13} \mathrm{C}$ NMR spectra for $\mathbf{1}$ and $\mathbf{5}$, HPLC for $\mathbf{5}$, and gels from radiometric kinase assays for $\mathbf{1}$ and $\mathbf{5}$ ). 
A derivative of the TBK1 inhibitor amlexanox (1) has been synthesized with deuterium introduced into two sites of metabolism on the C-7 isopropyl function. $d_{2}$-Amlexanox (5) displayed nearly equivalent potency to amlexanox ( $\mathrm{IC}_{50} 1.1 \mu \mathrm{M}$ vs $0.6 \mu \mathrm{M}$, respectively) against recombinant human TBK1. When incubated with human, rat, and mouse liver microsomes, 1 and $\mathbf{5}$ were stable $\left(\mathrm{t}_{1 / 2}>60\right.$ minutes) with 1 showing marginally greater stability relative to $\mathbf{5}$ except for rat liver microsomes.

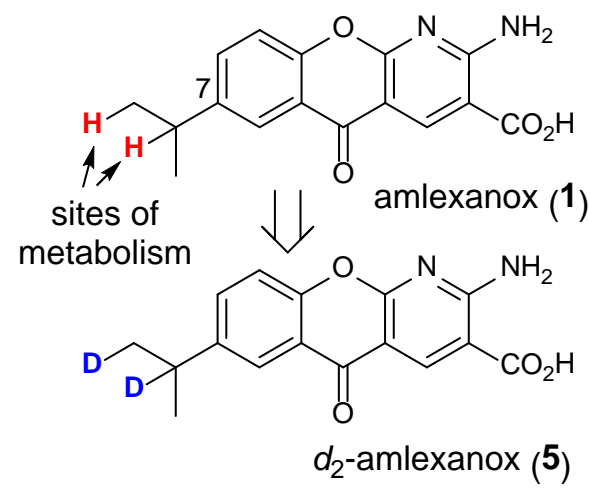

This article is protected by copyright. All rights reserved. 


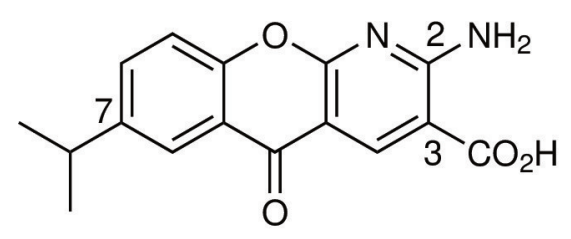

amlexanox

(1)

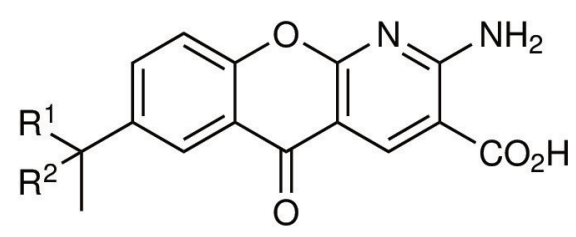

2: $\mathrm{R}^{1}=\mathrm{CH}_{3} ; \mathrm{R}^{2}=\mathrm{OH}$

3: $\mathrm{R}^{1}=\mathrm{CH}_{2} \mathrm{OH} ; \mathrm{R}^{2}=\mathrm{H}$

4: $\mathrm{R}^{1}=\mathrm{CO}_{2} \mathrm{H} ; \mathrm{R}^{2}=\mathrm{H}$<smiles>[2H]CC(C)(C)c1ccc2oc3nc(N)c(C(=O)O)cc3c(=O)c2c1</smiles>

jlcr_3716_f1.eps 


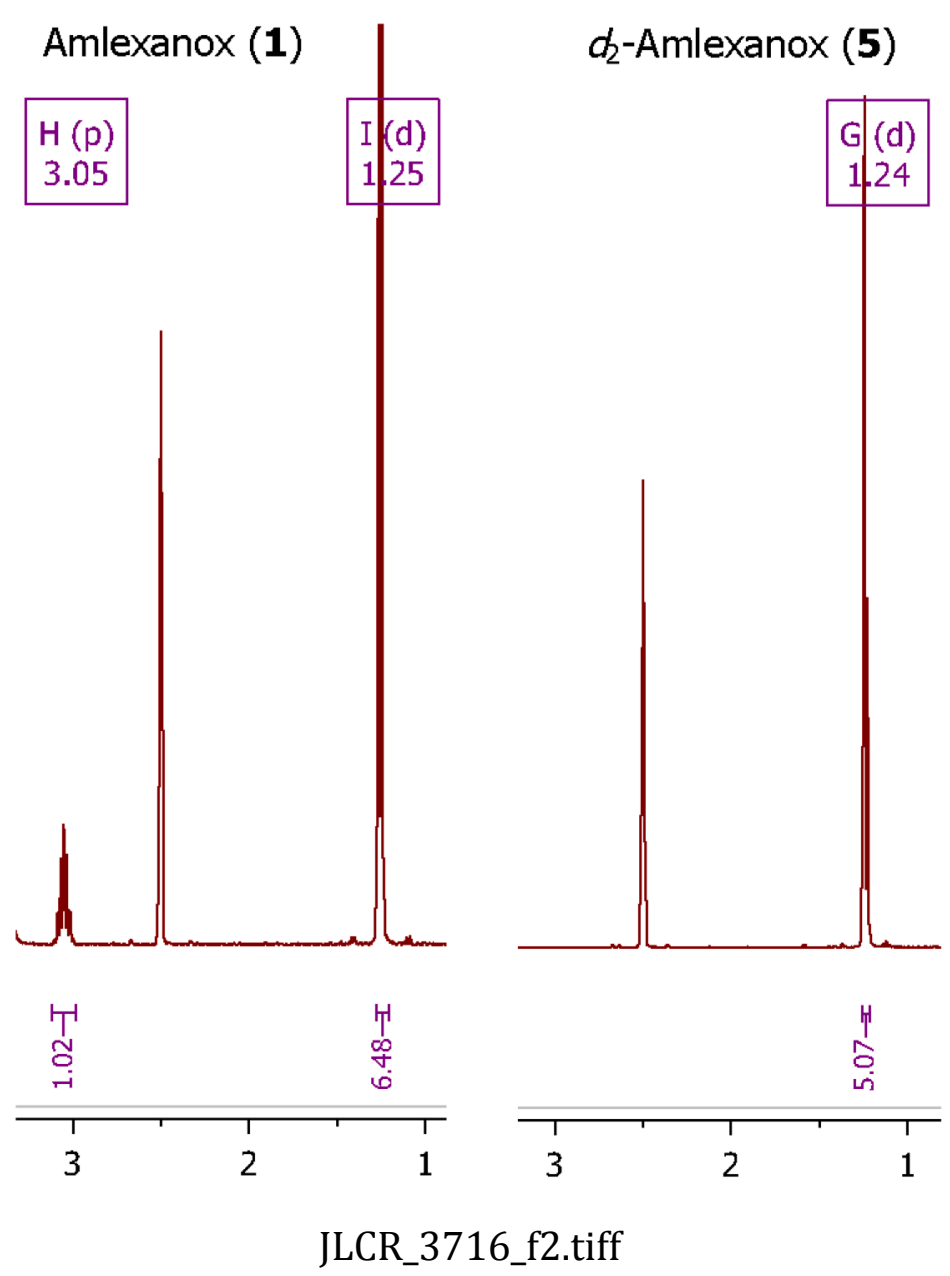

This article is protected by copyright. All rights reserved. 


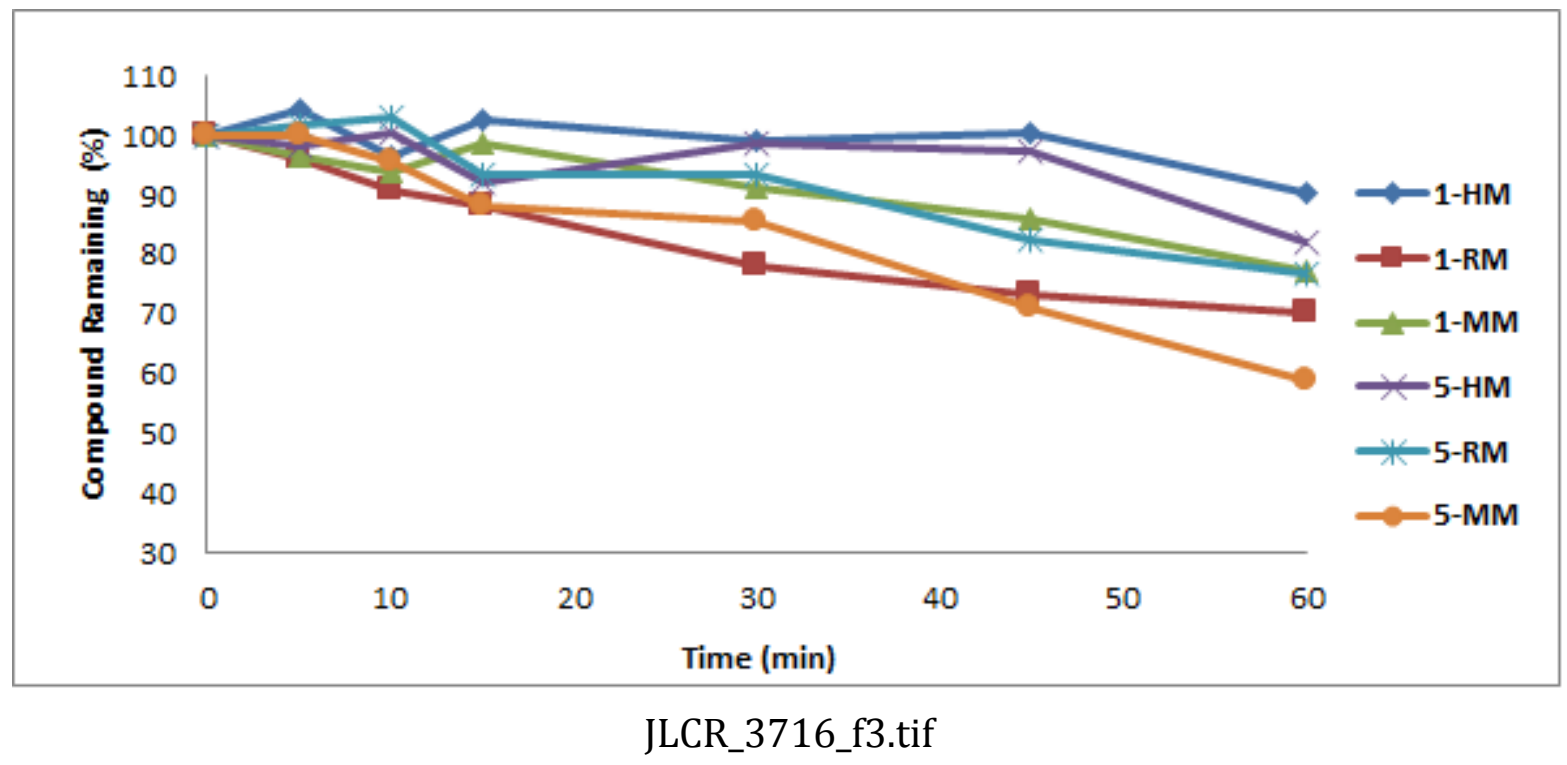

This article is protected by copyright. All rights reserved. 


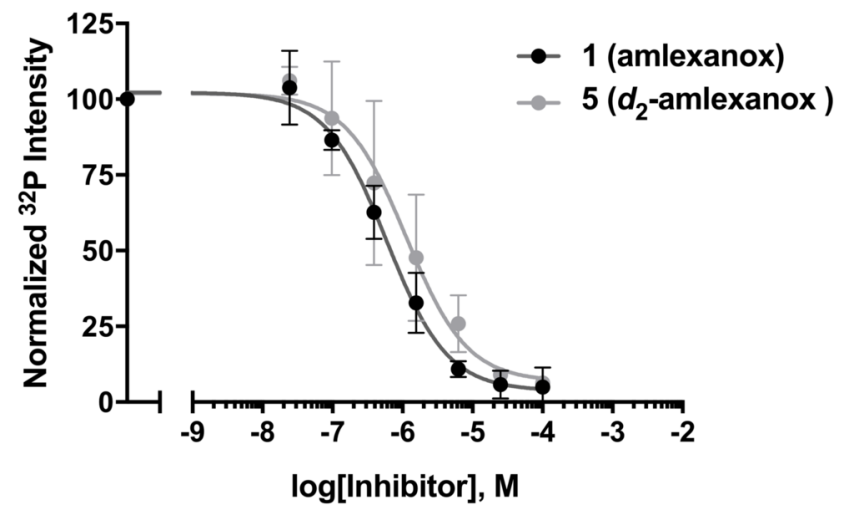

JLCR_3716_f4.tiff

This article is protected by copyright. All rights reserved. 

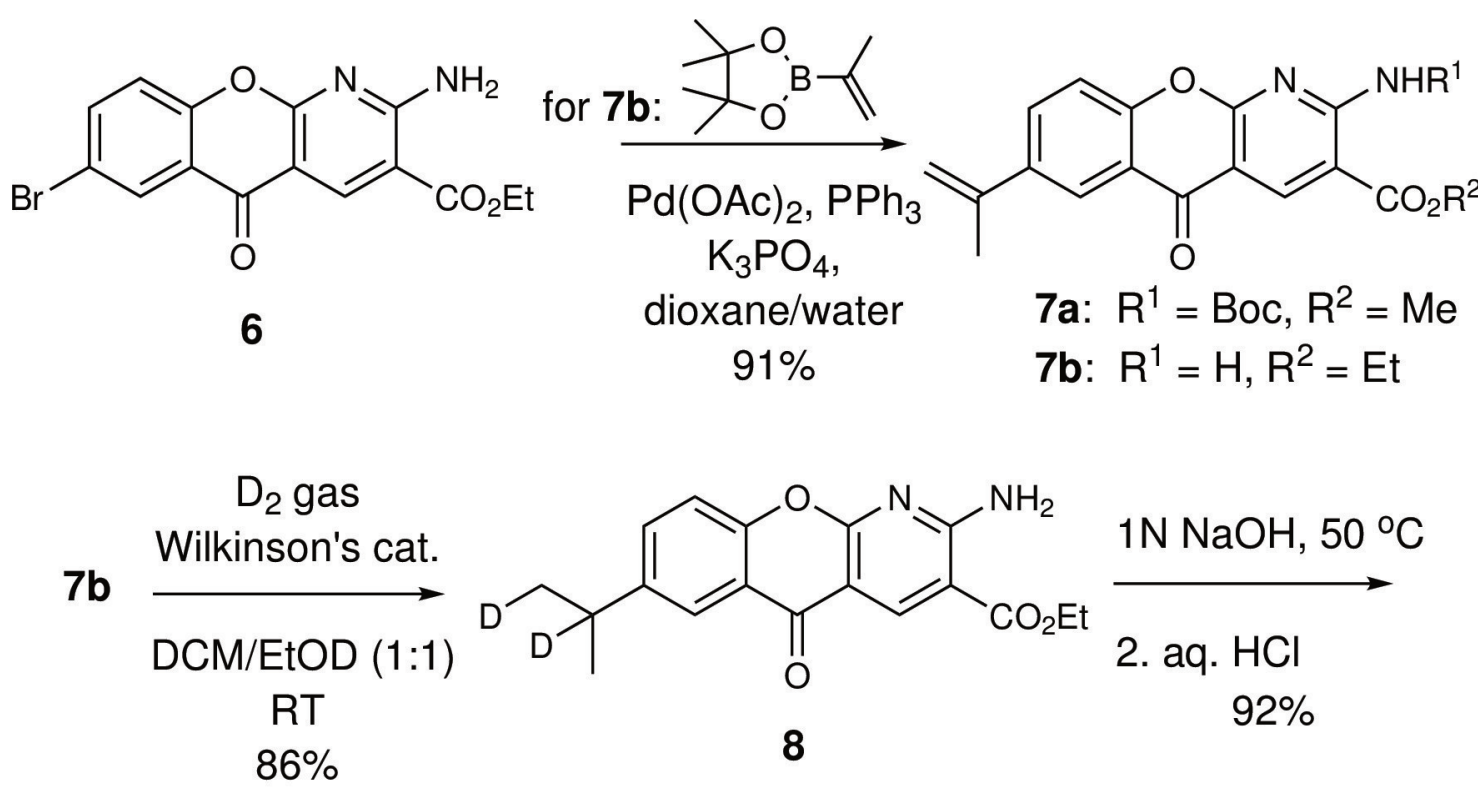<smiles>[2H]CC([2H])(C)c1ccc2oc3nc(N)c(C(=O)O)cc3c(=O)c2c1</smiles>

5

jlcr_3716_scheme 1.eps 